\title{
Representaciones sociales de futuros docentes de Física y de Química sobre el manual escolar
}

Social representations of prospective teachers of Physics and Chemistry about the textbook

\author{
Volumen 17, Número 2 \\ Mayo- Agosto \\ pp. 1-20
}

Este número se publica el $1^{\circ}$ de mayo de 2017

DOI: http://dx.doi.org/10.15517/aie.v17i1.28203

\author{
Carla Inés Maturano \\ Claudia Alejandra Mazzitelli
}

Revista indizada en REDALYC, SCIELO

Revista distribuida en las bases de datos:

LATINDEX, DOAJ, REDIB, IRESIE, CLASE, DIALNET, SHERPA/ROMEO, QUALIS-CAPES, MIAR

Revista registrada en los directorios:

ULRICH'S $, \underline{\text { REDIE}}, \underline{\text { RINACE}}, \underline{\text { OEI }}$ MAESTROTECA, PREAL, $\underline{\text { CLACSO }}$ 


\title{
Representaciones sociales de futuros docentes de Física y de Química sobre el manual escolar
}

Social representations of prospective teachers of Physics and Chemistry about the textbook

\author{
Carla Inés Maturano ${ }^{1}$ \\ Claudia Alejandra Mazzitelli ${ }^{2}$
}

\begin{abstract}
Resumen: El libro de texto de ciencias naturales es considerado uno de los recursos más utilizados para enseñar y aprender en estas disciplinas. Los contenidos mostrados y las actividades que se desarrollan en las clases, propuestos a menudo en los libros de texto, suelen estar alejados de los intereses del estudiantado. Por esto, resulta interesante indagar las representaciones que el futuro docente posee sobre este objeto social para vislumbrar características de sus prácticas futuras. En este artículo, presentamos los resultados de un estudio exploratorio con futuros profesores y profesoras, cuyo objetivo es caracterizar sus representaciones sociales acerca del libro de texto o manual escolar de ciencias naturales. Los supuestos teóricos y la metodología, consistente en una técnica de evocación y jerarquización, se sustentan en el marco de la teoría de las representaciones sociales. Los participantes en la investigación constituyen una muestra de ocho estudiantes universitarios del último año de los profesorados en Física y en Química de la Universidad Nacional de San Juan (Argentina). Los resultados obtenidos, desde un abordaje predominantemente cualitativo, han permitido encontrar el contenido y la estructura, como así también las actitudes asociadas a las representaciones en estudio, destacándose un excesivo apego al manual como recurso para el diseño de las actividades del aula. Estos resultados servirían para formular recomendaciones tendientes a optimizar la utilidad del manual en su futura práctica profesional, buscando trascender el modelo tradicional de enseñanza e incorporar elementos de otros modelos alternativos que promuevan el interés hacia el aprendizaje de las ciencias.
\end{abstract}

Palabras clave: representaciones sociales, manual escolar, ciencias naturales, Argentina

Abstract: The textbook of Natural Science is considered one of the most used resource for teaching and learning in these disciplines. The contents taught and the activities carried out in class, frequently proposed in textbooks, are often away from the interests of students. Therefore, it would be useful to investigate the representations that the prospective teacher has about this social object to highlight characteristics of their future practice. This paper presents the results of an exploratory study carried out with prospective teachers, aimed at characterizing social representations about the textbook of Natural Science. The theoretical assumptions and methodology, consisting in a technique of evocation and hierarchy, are based on the framework of the theory of Social Representations. Eight university students attending their final year of the professorship in Physics and Chemistry at the National University of San Juan (Argentina) constitute the sample. The results obtained, from a predominantly qualitative approach, have allowed us to find not only the content and the structure of the representations under study, but also the attitudes associated with them, highlighting an excessive attachment to the manual as a resource for designing classroom activities. These results would be useful for making recommendations about the textbook with the aim of optimizing its use in their future professional practice, going beyond the traditional teaching model and incorporating elements of other alternative models that promote interest in learning science.

Keywords: social representations, textbook, natural science, Argentina

${ }^{1}$ Profesora de la Facultad de Ciencias Exactas, Físicas y Naturales e investigadora en el Instituto de Investigaciones en Educación en Ciencias Experimentales (FFHA), Universidad Nacional de San Juan, Argentina. Especialista en docencia universitaria y Profesora en Física. Dirección electrónica: cmatur@ffha.unsj.edu.ar

2 Profesora Titular e investigadora del Instituto de Investigaciones en Educación en las Ciencias Experimentales de la Universidad Nacional de San Juan, Argentina. Investigadora del Consejo Nacional de Investigaciones Científicas y Técnicas (CONICET), Directora de proyectos sobre Enseñanza de las Ciencias Naturales. Doctora en Educación y Profesora en Física. Dirección electrónica: mazzitel@ffha.unsj.edu.ar

Artículo recibido: 29 de junio, 2016

Enviado a corrección: 8 de noviembre, 2016

Aprobado: 23 de enero, 2017 


\section{Introducción}

Para lograr una mayor motivación del alumnado y un aumento de su interés hacia el estudio de las ciencias naturales sería necesario que la enseñanza habitual se centre más en trabajos prácticos, relaciones entre Ciencia, Tecnología, Sociedad y Ambiente (CTSA), entre otros, que en aspectos más conceptuales y propedéuticos (Banet, 2007; Furió, Vilches, Guisasola y Romo, 2001). Esto implica que el profesorado asuma que la enseñanza de las ciencias en la escuela secundaria no está dirigida fundamentalmente a promover el acceso del grupo estudiantil a las carreras universitarias, sino que debería enfocarse en la formación de los futuros ciudadanos, lo que significaría no dar tanta prioridad al aprendizaje de los contenidos conceptuales frente a los actitudinales o procedimentales y, además, tener en cuenta los intereses de los estudiantes y de la sociedad (Furió et al., 2001). En este sentido, el panorama actual de la educación científica en la escuela secundaria resulta bastante preocupante porque enfatiza los aprendizajes memorísticos sobre contenidos de escasa relevancia personal y social para los no especialistas, mostrando una ciencia descontextualizada y aislada de la vida diaria, lo que pone de manifiesto el contraste existente entre la ciencia enseñada por los profesores y profesoras -que coincide con la que muestran los libros de texto- con la actual tecnociencia de la vida cotidiana (Banet, 2007).

Surge así la relevancia del análisis de la forma en la cual el docente o la docente propone usar en el aula el libro de texto, el cual es el recurso más utilizado para enseñar y aprender. Este, por un lado, una vez elegido por el profesorado recibe de su parte un alto grado de confianza, lo cual le otorga una gran responsabilidad en el proceso educativo, y por otro lado, el libro apuesta en escasas ocasiones por las innovaciones educativas porque las grandes editoriales consideran que así aseguran su aceptación y, por lo tanto, sus beneficios económicos (Solbes, Montserrat y Furió, 2007). Según Alzate, Arbeláez, Gómez, Romero y Gallon (2005), el libro de texto constituye una manera de intervenir en los procesos de aprendizaje del alumnado, pues es el docente quien cumple una función de organizador situado que presenta y organiza determinado tipo de saberes o destaca aquello que considera importante, moldeando así al texto escolar en cierto sentido, según las maneras, los conceptos, las prefiguraciones y situaciones de aprendizaje que el mismo docente ha constituido.

Suponemos que las representaciones sociales que un futuro docente tiene del uso de un libro de texto serán reflejadas en el modo en que planteará su utilización en el aula y en su futuro accionar durante las clases, impactando así en el aprendizaje e interés de los 
estudiantes. Esto justifica la necesidad de indagar cuáles son las representaciones de estudiantes del último año de profesorado en Física y en Química acerca del manual escolar usado para enseñar en las aulas de esas disciplinas.

\section{Breve referente teórico}

Esta investigación se encuadra en la propuesta teórica de Serge Moscovici para estudiar la construcción social de la realidad, conocida como teoría de las representaciones sociales. Moscovici (1979) define la representación social como una modalidad particular de conocimiento cuya función es la elaboración de comportamientos y la comunicación entre los individuos.

La representación de un grupo sobre una tarea define los objetivos y procedimientos específicos para sus miembros (Jodelet, 1986), tornándose en "una visión funcional del mundo que permite al individuo o al grupo conferir sentido a sus conductas, y entender la realidad mediante su propio sistema de referencias y adaptar y definir de este modo un lugar para sí" (Abric, 2001).

Una representación social está constituida por un conjunto organizado y estructurado de informaciones, creencias, opiniones y actitudes a propósito de un objeto dado, estando organizada alrededor de un núcleo central. Según Abric (2001), su estructura está conformada por:

- Núcleo: es el elemento fundamental que unifica y estabiliza la representación y que determina su significación y su organización. Tiene dos funciones esenciales: función generadora (crea y transforma la significación de los otros elementos constitutivos de la representación) y función organizadora (determina la naturaleza de los lazos que unen entre sí a los elementos restantes de la representación). Este núcleo central está constituido por uno o varios elementos y es el sector más estable de la representación, por lo que será el que más resistirá al cambio.

- Los elementos periféricos: se organizan alrededor del núcleo central y su presencia, su ponderación, su valor y su función están determinados por el núcleo. Están constituidos por informaciones retenidas, seleccionadas e interpretadas, juicios formulados al respecto del objeto y su entorno, estereotipos y creencias. Estos elementos están jerarquizados: los más próximos al núcleo desempeñan un papel importante en la concreción del significado de la representación y los más distantes del núcleo ilustran, aclaran y justifican esta significación. 
Según Mora (2002), las representaciones sociales pueden ser analizadas con fines didácticos y empíricos en tres dimensiones:

- La información: es la suma de conocimientos de un grupo con respecto a un acontecimiento, hecho o fenómeno de naturaleza social.

- El campo de representación: es la organización jerarquizada del contenido de la representación, que puede diferir de un grupo a otro e incluso en el interior de un mismo grupo.

- La actitud: es la orientación favorable o desfavorable con respecto al objeto de representación, que se constituye en el componente con más implicación comportamental y de motivación.

Jodelet (2008) destaca los estudios que consideran, desde esta perspectiva, la inscripción social de los sujetos y los grupos como organizadora de sistemas de significado y de representación relacionados con objetos de interés común. Dichos estudios se centran, entre otros, en las normas sociales e institucionales que orientan las prácticas de la educación. Por esto, como la enseñanza y el aprendizaje involucran procesos y acciones que superan lo individual, resulta conveniente abordarlas desde el enfoque psicosocial descripto. Piña y Cuevas (2004) expresan que el estudio de las representaciones sociales de los actores de la educación nos permite conocer el interior de las escuelas y el sentido que en ellas se adjudica a los procesos educativos.

Centrar el foco de análisis en la formación inicial del futuro docente, en sus actividades y vivencias cotidianas, en cómo los estudiantes van construyendo modos de pensamiento y comprensión permitirá interpretar sus futuras prácticas (D’Andrea y Corral de Zurita, 2006). Sancho et al. (2014) hallaron que en las prácticas docentes durante los primeros años de trabajo subyace más la inercia y lo que siempre se ha hecho, que el conocimiento y los tipos de enseñanza basados en lo que hoy se sabe acerca de cómo aprenden los estudiantes. Es decir, en los primeros momentos, los docentes en general tienden a recurrir más a prácticas aprendidas en su larga experiencia como alumnos que a la enseñanza recibida en la universidad, influyendo así en las prácticas del aula los valores y principios construidos social y culturalmente en un entramado complejo de significaciones que cruzan las instituciones y que forman parte del modo como se entienden determinados procesos educativos (Rivas y Leite, 2013).

Los docentes noveles de ciencias de escuela primaria, al comenzar su práctica profesional, plantean actividades de búsqueda de información, trabajo con material concreto 
y experimentación, pero evidencian dificultades para abordar la enseñanza desde la perspectiva de la ciencia escolar (Ibaceta, Moscato y Schnersch, 2012). Solís, Luna y Rivero (2002) encontraron que los profesores principiantes de ciencias naturales que se incorporan a la educación secundaria no han desarrollado recursos profesionales apropiados en muchos aspectos, por lo que sus ideas sobre la enseñanza no son muy diferentes a las del conocimiento cotidiano más generalizado. Estos autores consideran que la formación inicial de los docentes debería promover un cambio, tanto conceptual como metodológico, en las concepciones sobre la docencia, pues la mayoría de los futuros profesores se mueven en un modelo didáctico tradicional, por ser generalmente el único referente visto en su etapa como estudiantes.

Así como el alumnado llega a las clases de ciencias con ideas sobre los contenidos que va a estudiar, el cuerpo docente también se inicia en la docencia con creencias sobre la enseñanza y el aprendizaje de las ciencias, que influyen en su quehacer (García-Carmona, 2013; Vaillant, 2007). Las representaciones sociales de los docentes sobre la enseñanza, el aprendizaje y sus alumnos se relacionan con las características de su práctica (Guirado, Mazzitelli y Olivera, 2013; Marcelo y Vaillant, 2009; Mazzitelli, Aguilar, Guirado y Olivera, 2009; Mazzitelli, 2015; entre otros). En consecuencia, las representaciones sociales de los estudiantes de profesorado en ciencias definirán los objetivos y las estrategias específicas que plantearán en sus clases, por lo que el estudio de las representaciones sociales de los sujetos que intervienen en el área de la educación científica permitirá conocer la forma en que interpretan y construyen su conocimiento sobre la ciencia y sobre los procesos y tareas educativas en esta área, para poder formular propuestas que permitan mejorar las prácticas.

Se han abordado, hasta el momento, en diversos estudios, las representaciones sociales de los estudiantes de profesorado acerca de la ciencia (Melo, Tenório y Accioly, 2010), la física (Zorrilla y Mazzitelli, 2014), la química (Cortes Junior, Corio y Fernandez, 2010); la física, la química y la docencia (Mazzitelli, 2012); la docencia (Mazzitelli, Guirado y Olivera, 2010), ser profesor (Lima Miranda, 2014; Santos de Jesus, Nunes, Santos de Jesus y Pagan, 2011), la enseñanza de las ciencias (Mazzitelli, 2013), y las actitudes relacionadas con el aprendizaje de las ciencias (Guirado, Mazzitelli y Olivera, 2012), pero no hemos encontrado, haciendo un exhaustivo análisis, estudios que indaguen acerca de las representaciones sociales acerca del uso del manual escolar o libro de texto de ciencias naturales, aun cuando este se considera como uno de los recursos más utilizados para enseñar en este ámbito disciplinar en diversos contextos educativos (Cuéllar, Pérez y Quintanilla, 2005; García y Moro, 2004; Guerra y López, 2011; Martínez y García, 2003). 
Los manuales escolares se consideran una clase textual caracterizada por su impronta pedagógica que alude a su carácter de libro "fácil de entender", lo cual implicaría que los contenidos están presentados allí de una forma fácil de abordar, por lo que deben estar organizados y presentados con claridad (Gazali, 2005). Los manuales escolares reciben diferentes denominaciones que usaremos indistintamente en este artículo, las cuales varían según los países, llamándose comúnmente "libro de texto" en Argentina o España, "texto escolar" en Colombia, "libro didáctico" (livro didático, en portugués) en Brasil, entre otras (Tosi, 2011).

La mayoría de los trabajos realizados en el ámbito latinoamericano sobre los libros de texto han privilegiado temáticas como la problemática del género, el racismo, la discriminación religiosa o la formación de estereotipos vinculados a crear identidades colectivas, los cuales no agotan todas las posibilidades de investigación sobre el libro de texto (Ramírez, 2003). Este autor destaca la necesidad de abrir el espectro investigativo sobre esta fuente primaria, analizando, por ejemplo, su uso por parte de los docentes, partiendo de que, aunque no todos ellos usan los libros de texto de la misma manera en el aula, "es necesario detectar aquellas variables que dan sentido a tales actuaciones, algunas de ellas de carácter personal, otras de carácter académico, institucional, pedagógico, ideológico, religioso o político, que podrían servir para explicar la conducta de los maestros con respecto al texto escolar" (Ramírez, 2003, p. 288).

En el ámbito específico de las ciencias naturales, se han podido identificar los métodos y técnicas utilizados habitualmente por los docentes, evidenciando que están muy apegados a la herencia dejada por el positivismo (Torres, 2010). La dependencia del libro de texto es tal que se considera que la principal forma de enseñar ciencias es como "un conjunto de hechos y verdades estables e incuestionables, que el libro de texto contiene, que el profesor, supuestamente, sabe y que el alumno tiene que memorizar, para poder contestar las preguntas a las que es sometido en los exámenes" (Torres, 2010, p. 135).

La teoría de las representaciones sociales se constituye en un punto de vista adecuado para analizar las representaciones acerca del manual escolar, puesto que en su utilización para la enseñanza y el aprendizaje se intersectan lo psicológico y lo social. Considerando las ideas de Jodelet (1986) y aplicándolas a esta investigación, son los docentes, sujetos sociales, los que aprehenden en la vida diaria un conocimiento de sentido común del uso de un texto para enseñar y aprender a partir de sus experiencias, de informaciones, conocimientos y modelos de pensamiento que cada uno recibe y transmite a través de la tradición, la educación y la comunicación social. Así, el conocimiento acerca de qué, cómo y 
para qué se usa el manual en la clase de ciencias naturales es un conocimiento práctico, socialmente elaborado y compartido. Por esto, consideramos necesario conocer las representaciones sociales de los futuros docentes acerca del libro de texto utilizado para enseñar esta materia para así comprender algunas acciones y actitudes relacionadas con la enseñanza y el aprendizaje de estas disciplinas. Analizando dichas representaciones, sería posible proponer hipótesis sobre cuáles elementos podrían obstaculizar y cuáles favorecer el aprendizaje y el interés por las ciencias.

En este artículo, analizaremos dichas representaciones en sus tres dimensiones, considerando: los elementos informativos que la muestra tiene en relación con el manual escolar usado para enseñar, el campo de representación o estructura jerárquica de dichos elementos y la actitud hacia este objeto de representación. En este caso, la primera dimensión (información) conduce necesariamente a los datos y explicaciones que se forman los sujetos acerca del uso del manual escolar en sus relaciones cotidianas de enseñar y aprender ciencias naturales; la segunda dimensión (campo de representación) a la organización de dichos elementos; y la tercera dimensión (actitud) a la posición que toman los futuros docentes frente al manual escolar como objeto de representación.

\section{Metodología}

La muestra de estudiantes de profesorado seleccionada para este estudio exploratorio está constituida por 8 estudiantes que participaron voluntariamente de la investigación. Estos constituyen el $67 \%$ de la población total de estudiantes del último año de los profesorados en Física y en Química de la Universidad Nacional de San Juan (Argentina). Mientras participó en este estudio, el total de los estudiantes estaba realizando su práctica de la enseñanza en el nivel medio y había terminado de cursar las materias de la carrera o cursaba algunas materias del último año.

Para identificar el contenido y la estructura de una representación social conviene utilizar métodos y técnicas en las que los sujetos efectúen un trabajo cognitivo de análisis, comparación y jerarquización de su propia producción (Abric, 2001). Una forma de hacerlo sería, según este autor, pedir asociaciones libres y espontáneas, a partir de un término inductor, para que el sujeto produzca todos los términos, expresiones o adjetivos que se le presenten al espíritu, a fin de acceder de este modo a los elementos que constituyen el universo semántico del término o del objeto estudiado.

Así, el abordaje metodológico seleccionado para este estudio consiste en una técnica de asociación de palabras, llamada técnica de evocación y jerarquización, para lo cual le 
solicitamos a los estudiantes de profesorado que mencionaran cinco palabras que asociaran con el manual escolar de ciencias naturales utilizado para enseñar y les asignaran un orden de importancia. La cantidad de palabras solicitada ya ha sido utilizada en otros estudios y su elección se basa en que varias investigaciones muestran que, al emplear este tipo de técnicas, los participantes mencionan espontáneamente hasta cinco palabras (Abric, 2001; Chiavone, 2014; Mazzitelli et al., 2009; Petracci y Kornblit, 2007).

La técnica se implementó al comienzo de un taller, con una duración aproximada de tres horas reloj, en el que participaron los estudiantes de profesorado. Al comenzarlo, se les solicitó que escribieran cinco palabras o ideas breves que asociaban con el objeto de representación "manual escolar de ciencias naturales" y le asignaran una jerarquía. Posteriormente, elaboraron un plan de clase en el marco de las actividades propuestas en el taller (cuyo análisis escapa del objetivo de este artículo). Finalmente, revisaron las cinco palabras o frases escribiendo en algunos casos su relación con la tarea de planear la clase en la que se usa el manual escolar.

\section{Resultados y su análisis}

A partir de las palabras e ideas relevadas en este estudio, elaboramos seis categorías para agruparlas y ordenarlas: Características generales, Secciones, Recurso, Procesos educativos, Modos de trabajo en el aula y Estrategias. En las Tablas 1, 2 y 3 mostramos la definición de cada una de estas categorías, las subcategorías que incluye, si corresponde, y un ejemplo de palabra o idea breve para cada una, acompañando con la cita correspondiente de la relación planteada por el estudiante con respecto al plan de clase en aquellos casos en los cuales los estudiantes fundamentaron sus expresiones. Para la identificación de cada estudiante de la muestra, se ha indicado al final de cada ejemplo un número de orden asignado al azar (del 01 al 08), seguido de otro número que indica el orden de importancia asignado a la palabra o idea breve citada (del 10 más importante al 50 menos importante).

La asignación de cada idea a una determinada categoría ha sido definida por consenso entre dos investigadores, teniendo en cuenta el concepto o idea evocado por cada estudiante y, para decidir ambigüedades, además de su interpretación directa, se ha considerado la explicación dada por el estudiante en relación con el plan de clase elaborado por él mismo. 
A partir de las categorías ya enunciadas, para profundizar el análisis, las agrupamos en dimensiones (Graça, Moreira y Caballero, 2004; Mazzitelli, 2007), según atiendan a: el manual en sí mismo, las potencialidades del manual y el manual en acción, respectivamente. Las dimensiones elaboradas y los criterios considerados son:

- Dimensión intrínseca del género manual: incluye las categorías de Características generales y Secciones. Esta dimensión se refiere específicamente a "qué" aspectos propios del género textual manual escolar forman parte de las representaciones sociales.

- Dimensión funcional del manual: incluye las categorías de Recurso y Procesos educativos. Esta dimensión se relaciona con "para qué" se propone el uso del manual escolar.

- Dimensión metodológica del manual: incluye las categorías de Estrategias y Modos de trabajo. Esta dimensión se asocia a "cómo" se propone utilizar el manual.

Tabla 1

Categorías, subcategorías y ejemplos de la dimensión intrínseca DIMENSIÓN INTRÍNSECA DEL GÉNERO MANUAL

\begin{tabular}{|c|l|}
\hline \multicolumn{1}{|c|}{ DIMENSIÓN INTRÍNSECA DEL GÉNERO MANUAL } \\
\hline Categorías & \multicolumn{1}{|c|}{ Definición, subcategorías y ejemplos } \\
\hline \multirow{5}{*}{$\begin{array}{c}\text { Características } \\
\text { generales }\end{array}$} & $\begin{array}{l}\text { Hace referencia a aspectos comunes del género manual o de este tipo de libros y } \\
\text { sus valoraciones generales, como son: } \\
\text { características propias del género manual. Ejemplo: lenguaje (03-3), refiriéndose al } \\
\text { lenguaje "escolar". }\end{array}$ \\
especificaciones o características comunes a cualquier texto académico o escolar. \\
& $\begin{array}{l}\text { Ejemplo: accesible en el costo (05-3), indicando que "Se puede tomar como libro de } \\
\text { texto para el curso y, dependiendo del nivel económico del alumnado, (...)". } \\
\text { valoraciones de las características generales. Ejemplo: no muy completo (05-4), } \\
\text { "(...) porque el docente debe buscar otra bibliografía para encargar una tarea de } \\
\text { investigación". }\end{array}$ \\
\hline $\begin{array}{c}\text { Incluye palabras e ideas que se refieren directamente a partes o secciones del } \\
\text { manual escolar. Las siguientes subcategorías responden a las secciones } \\
\text { mencionadas pero de ningún modo agotan las secciones existentes en los } \\
\text { manuales escolares actuales, a saber: } \\
\text { Contenidos desarrollados (explicitados generalmente en el índice o al comienzo de } \\
\text { cada capítulo y tratados en profundidad mediante textos expositivo-explicativos). } \\
\text { Ejemplo: información (01-2), agregando que se refiere a la "información que utiliza } \\
\text { el docente para armar la clase y seleccionar los temas". } \\
\text { Actividades (incluidas de diversos modos en el manual). Ejemplo: actividad (04-5). } \\
\text { Imágenes (intercaladas con los textos en casi la totalidad de las secciones del } \\
\text { manual). Ejemplo: imágenes (07-2), indicando que "marco la observación de las } \\
\text { imágenes que acompañan al texto y se hace pensar a los alumnos antes de la } \\
\text { lectura (...)". } \\
\text { Experimentos: incluidos con diferentes grados de complejidad y apertura en los } \\
\text { manuales escolares del área. Ejemplo: prácticas (07-3), indicando que se refiere } \\
\text { así a "(...) práctica de laboratorio o experiencia". }\end{array}$ \\
\hline
\end{tabular}

Fuente: Elaboración propia de las autoras con base en resultados de la técnica aplicada con estudiantes de profesorado Universidad Nacional de San Juan (2016). 
Tabla 2

Categorías, subcategorías y ejemplos de la dimensión funcional

\section{DIMENSIÓN FUNCIONAL DEL MANUAL}

\begin{tabular}{|c|l|}
\hline \multicolumn{2}{|c|}{ DIMENSIÓN FUNCIONAL DEL MANUAL } \\
\hline Categorías & \multicolumn{1}{|c|}{ Definición, subcategorías y ejemplos } \\
\hline & $\begin{array}{l}\text { Incluye palabras e ideas que consideran al manual como recurso, en el sentido en } \\
\text { que se constituye en un medio o instrumento que le facilita la tarea a alguien y le } \\
\text { permite conseguir los objetivos que pretende. Según las explicaciones, en las que } \\
\text { los futuros docentes han especificado quién es ayudado por el manual, es posible } \\
\text { subdividir esta categoría en las siguientes subcategorías: }\end{array}$ \\
$\begin{array}{l}\text { Recurso } \quad \text { Recurso para el docente. Ejemplo: ayuda docente (08-4), porque "Ayuda en } \\
\text { mi plan a la transposición didáctica, enseñanza-aprendizaje, selección de textos } \\
\text { escolares, etc.". }\end{array}$ \\
$\begin{array}{l}\text { Recurso para el alumno. Ejemplo: útil (05-1), indicando posteriormente que } \\
\text { "Porque es lo que las bibliotecas tienen y los alumnos pueden consultar (...)". }\end{array}$ \\
$\begin{array}{l}\text { Recurso para ambos (docente y alumno). Ejemplo: herramientas (01-1), } \\
\text { señalando explícitamente que se trata de "Herramientas para el alumno y para el } \\
\text { docente". }\end{array}$ \\
\hline $\begin{array}{l}\text { Procesos } \\
\text { educativos }\end{array}$ & $\begin{array}{l}\text { Incluye palabras e ideas referidas a los procesos que se producen en el aula, } \\
\text { mencionados en forma general. Ejemplo: aprendizaje (01-5). }\end{array}$ \\
\hline
\end{tabular}

Fuente: Elaboración propia de las autoras con base en resultados de la técnica aplicada con estudiantes de profesorado Universidad Nacional de San Juan (2016).

Tabla 3

Categorías, subcategorías y ejemplos de la dimensión metodológica

\begin{tabular}{|c|l|}
\hline \multicolumn{2}{|c|}{ DIMENSIÓN METODOLÓGICA DEL MANUAL } \\
\hline $\begin{array}{c}\text { Categorías } \\
\text { Modos de } \\
\begin{array}{c}\text { trabajo en el } \\
\text { aula }\end{array}\end{array}$ & $\begin{array}{l}\text { Encierinición, subcategorías y ejemplos } \\
\text { trabajo en el aula usando el manual. Ejemplo: grupos (06-2). }\end{array}$ \\
\hline Estrategias & $\begin{array}{l}\text { Incluye tareas mencionadas en forma específica que usan el manual y podrían } \\
\text { proponerse en diferentes momentos de la clase. Ejemplo: guía de preguntas } \\
\text { (cuestionario) (06-3), explicando que "Es importante para que los alumnos presten } \\
\text { más atención al texto y le queden conceptos más claros". }\end{array}$ \\
\hline
\end{tabular}

Fuente: Elaboración propia de las autoras con base en resultados de la técnica aplicada con estudiantes de profesorado Universidad Nacional de San Juan (2016).

Para procesar los datos obtenidos, teniendo en cuenta las categorías y dimensiones elaboradas, analizamos la frecuencia para cada una de ellas y el orden de importancia asignado, siguiendo un modo de procesamiento similar al utilizado por Mazzitelli et al. (2009) y Chiavone (2014). De esta forma, establecemos si la frecuencia de cada categoría es alta o baja y si la importancia asignada a esta es grande o pequeña de la siguiente manera:

- Consideramos que la frecuencia de cada categoría es alta o baja teniendo en cuenta las frecuencias absolutas de todas las categorías; calculamos el promedio entre la mayor y la menor frecuencia de las categorías y lo comparamos con la frecuencia de cada categoría: si tiene mayor frecuencia que el valor promedio, decimos que es de frecuencia alta y si tiene menor, decimos que su frecuencia es baja. 
- Consideramos que la importancia de cada categoría es grande o pequeña comparando con el promedio de las ponderaciones de cada una de las palabras que la integran. A las palabras mencionadas en primer lugar les asignamos el valor 1; a las mencionadas en segundo lugar, el 2; y así sucesivamente hasta alcanzar el 5 para las mencionadas en quinto y último lugar. Para cada palabra, consideramos el orden en que fue mencionada por cada uno de los estudiantes de la muestra. La importancia es grande (Graça, Moreira y Caballero 2004; Mazzitelli et al., 2009; Wachelke y Wolter, 2011) cuando el valor del promedio para la categoría es menor que 3 y la importancia es pequeña cuando el promedio es mayor o igual a 3.

La Tabla 4 muestra las categorías ordenadas según su frecuencia y la Tabla 5 según su importancia. El promedio entre la menor y mayor frecuencia es 6, por lo que las categorías Secciones, Características generales, Estrategias y Recurso, cuya frecuencia es mayor que 6, resultan de frecuencia alta; y las categorías Procesos educativos y Modo de trabajo en el aula resultan de frecuencia baja. En lo que se refiere a la importancia, las categorías Modo de trabajo en el aula, Estrategias y Recurso resultan de importancia alta y las categorías Secciones, Características generales y Procesos educativos de importancia baja.

Tabla 4

Categorías en orden decreciente de frecuencia

\begin{tabular}{|l|c|}
\hline \multicolumn{1}{|c|}{ Categorías } & \multicolumn{1}{|c|}{$\begin{array}{c}\text { Frecuencia } \\
\text { Absoluta }\end{array}$} \\
\hline Secciones & 11 \\
\hline Características generales & 9 \\
\hline Estrategias & 8 \\
\hline Recurso & 8 \\
\hline Procesos educativos & 3 \\
\hline Modo de trabajo en el aula & 1 \\
\hline
\end{tabular}

Fuente: Elaboración propia de las autoras con base en resultados de la técnica aplicada con estudiantes de profesorado Universidad Nacional de San Juan (2016).

Tabla 5

Categorías en orden decreciente de importancia

\begin{tabular}{|l|c|}
\hline \multicolumn{1}{|c|}{ Categorías } & Importancia \\
\hline Modo de trabajo en el aula & 2 \\
\hline Estrategias & 2,63 \\
\hline Recurso & 2,63 \\
\hline Secciones & 3 \\
\hline Características generales & 3,44 \\
\hline Procesos educativos & 4 \\
\hline
\end{tabular}

Fuente: Elaboración propia de las autoras con base en resultados de la técnica aplicada con estudiantes de profesorado Universidad Nacional de San Juan (2016). 
En función de los valores obtenidos para la frecuencia y la importancia de cada categoría, podemos identificar las cuatro zonas características de las representaciones sociales en estudio:

- Núcleo: frecuencia alta - gran importancia

- Primera periferia: frecuencia alta - importancia pequeña

- Elementos de contraste: frecuencia baja - gran importancia

- Segunda periferia: frecuencia baja - importancia pequeña

La Figura 1 muestra la estructura de las representaciones en estudio.

Figura 1

\section{Estructura determinada para las representaciones sociales acerca del manual escolar de ciencias naturales}

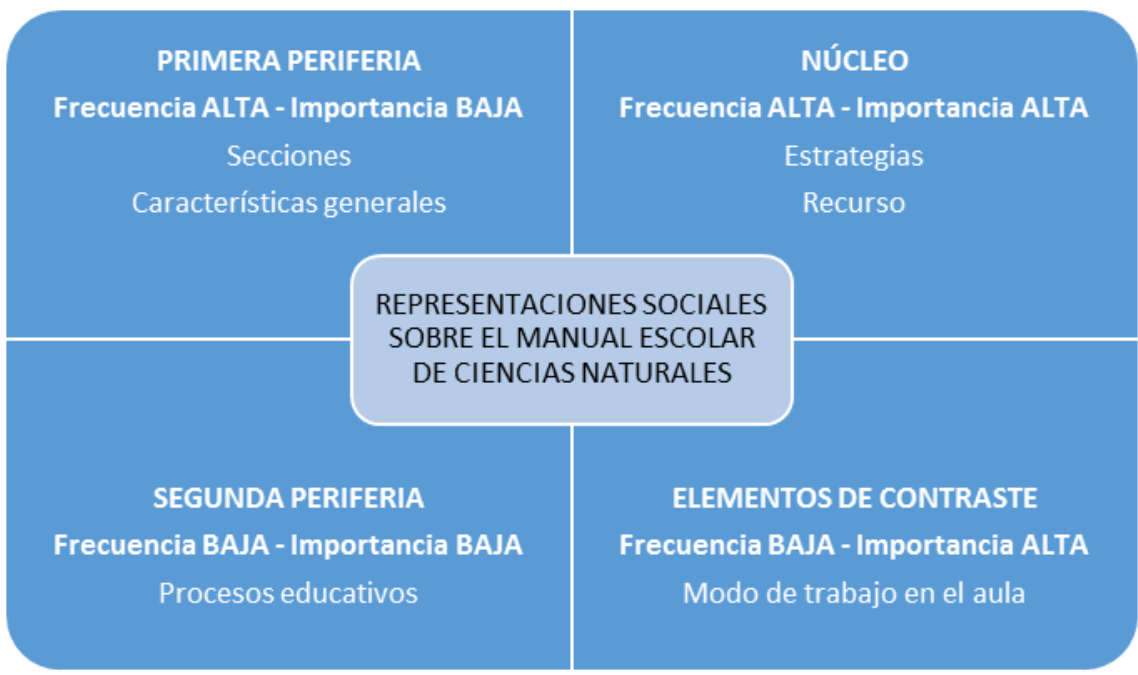

Fuente: Elaboración propia de las autoras con base en resultados de la técnica aplicada con estudiantes de profesorado Universidad Nacional de San Juan (2016).

Del análisis de la estructura hallada para las representaciones sociales en estudio, puede inferirse que en el núcleo aparecen las categorías Estrategias y Recurso, asociadas a las dimensiones Metodológica y Funcional del manual escolar de ciencias naturales, con frecuencia e importancia que toman igual valor para ambas categorías. Esto indicaría que las RS de los estudiantes de profesorado aparecen vinculadas principalmente con las tareas específicas a las que asocian el uso del manual, como la lectura, y con la función misma que el manual desempeña como herramienta tanto para la enseñanza como para el aprendizaje. En la Figura 2 se explicitan las palabras e ideas recolectadas en estas categorías que integran el núcleo de las representaciones. 
Figura 2

Conceptos e ideas relevadas para las categorías que forman parte del núcleo de las representaciones sociales en estudio

\begin{tabular}{ll}
\multicolumn{1}{c}{ Categoria Estrategias } & \multicolumn{1}{c}{ Categoría Recurso } \\
& \\
\hline -Síntesis & - Transposición didáctica \\
- Leer el siguiente párrafo & -Apoyo \\
-Actividad extraescolar & -Ayuda docente \\
- Elaborar un proyecto & -Útil \\
- Lectura comprensiva & - Contacto con el alumno \\
- Guía de preguntas & -Herramientas \\
(cuestionario) & -A disposición \\
- Lectura & -Guía \\
- Observar la imagen &
\end{tabular}

Fuente: Elaboración propia de las autoras con base en resultados de la técnica aplicada con estudiantes de profesorado Universidad Nacional de San Juan (2016).

Por una parte, del análisis de las ideas que forman parte de la categoría Estrategias, notamos que se refieren a actividades bastante ligadas a la enseñanza tradicional en la mayoría de los casos. Por otra parte, las ideas de la categoría Recurso se asocian al texto usado como herramienta, lo cual corrobora los supuestos respectos del protagonismo que tiene su uso sistemático por parte del alumno y del docente en las prácticas áulicas.

En la primera periferia surgen categorías que refuerzan el núcleo en lo que se refiere a la Dimensión Intrínseca del género textual, agrupándose en esta zona los aspectos que definen y describen al manual en sí mismo; y en la segunda periferia aparece la categoría Procesos, incluida en la Dimensión Funcional, lo que muestra que es baja la importancia y la frecuencia con que los estudiantes de profesorado relacionan el manual con los procesos educativos generales en que se enmarca su uso. El Modo de trabajo en el aula forma parte de la zona de los elementos de contraste, mostrando así la importancia que tiene este aspecto para una minoría, con una frecuencia poco significativa en esta investigación.

Para completar el análisis en relación con las actitudes que forman parte de las representaciones sociales en estudio, examinamos las palabras o ideas que suponen una toma de posición frente al objeto de representación, las cuales podrían estar incluidas en cualquiera de las dimensiones analizadas anteriormente para determinar la estructura. De este modo encontramos:

- Actitudes favorables: hacen referencia a aspectos valorizados del manual, correspondiendo a actitudes positivas que señalan una buena disposición hacia el 
manual, como son: accesible en el costo (05-3); apoyo (08-3); ayuda docente (08-4); útil (05-1); herramientas (01-1); a disposición (07-4); guía (08-5).

- Actitudes desfavorables: hacen referencia a aspectos desvalorizados del manual, que se relacionan con actitudes negativas que indican una mala disposición señalando características negativas del manual escolar, como son: mínimos (03-5) ya que "No sólo el manual facilita la planificación.”; no muy completo (05-4), “(..) porque el docente debe buscar otra bibliografía para encargar una tarea de investigación".

- Actitudes críticas: consideran tanto aspectos positivos como negativos del objeto de representación, como por ejemplo: tiene temas variados (05-5) porque “(...) al ser multitemas, no tienen demasiada profundidad". En este caso, se valora la variedad como positiva, pero se destaca que, asociado a esto, se pierde en profundidad de cada tema.

\section{Conclusiones}

Los resultados obtenidos nos ayudan a verificar las hipótesis con respecto a las representaciones de los estudiantes de profesorado acerca del uso del manual escolar de ciencias, especialmente en lo que se refiere a su protagonismo en las prácticas áulicas y al uso de este a través del planteo de estrategias bastante ligadas a un modelo tradicional de enseñanza. Además, el estudio realizado nos ha permitido caracterizar mejor el conocimiento del manual escolar que los estudiantes han construido socialmente para poder delinear intervenciones que tiendan a revertir los obstáculos hallados, mediante acciones que involucren tanto la formación inicial como la formación continua de los docentes en un futuro próximo.

El contenido y la estructura detectados nos permiten revisar la organización jerárquica de los diversos elementos de las representaciones estudiadas. En el núcleo de dichas representaciones resalta la utilidad del manual, ya sea expresada en forma general indicando que se trata de un recurso, una herramienta o una guía, o en forma más específica, resaltando las tareas o estrategias que propondrían los futuros docentes en el aula a partir del manual escolar. La relevancia detectada para las dimensiones funcional y metodológica del manual como recurso y fuente para el diseño de las actividades en el aula de ciencias llevaría a pensar en un rol excesivamente predominante en las representaciones. La centralidad de estos elementos mostraría que para los estudiantes de profesorado de la muestra, cuando están recorriendo la etapa final de su formación inicial, el manual se 
transforma principalmente en el recurso que les ayuda a organizar sus propuestas áulicas concretas.

Los rasgos intrínsecos del manual, sus características y secciones, como elementos de la primera periferia, asumen un papel menos relevante que los anteriores, pero son importantes en la concreción del significado de la representación. Los de la segunda periferia, más distantes del núcleo, ilustran, aclaran y justifican esta significación, enmarcando los elementos anteriores en relación con los procesos educativos generales.

Las actitudes positivas hacia el uso del manual escolar, que se mencionan en mayor medida que las otras, no son necesariamente "positivas" para favorecer el interés y podrían estar marcando un excesivo apego al manual como único recurso para enseñar y aprender ciencias. En la búsqueda de una superación del modelo tradicional de enseñanza, sería más valorada una actitud crítica que considere que el manual tiene aspectos que necesitan ser cuestionados y/o mejorados. Esta característica de las representaciones sociales debería llevar a revisar el uso del manual como recurso didáctico exclusivo para plantear la necesidad de elaborar materiales didácticos alternativos, que se salgan de la rutina educativa habitual en la enseñanza de las ciencias. (García-Carmona, 2013)

Si relacionamos la importancia que le dan los estudiantes al manual en sus representaciones sociales con el hecho de que no han sido preparados en su formación inicial para hacer un uso intensivo y eficiente de este recurso y para proponer, a partir de la lectura, tareas que favorezcan el aprendizaje disciplinar (Lotti de Santos, Salim, Raya y Dori, 2008; Soliveres, Maturano y Quiroga, 2014), es posible vislumbrar que en su práctica docente futura hagan un uso intuitivo que responda solo a concepciones de sentido común acerca la lectura, en vez de plantear una propuesta que integre sus representaciones con las recomendaciones actuales de las investigaciones en enseñanza de las ciencias naturales.

Como el núcleo contiene los elementos más estables de las representaciones, los resultados hallados, unidos al hecho de una mayor cantidad de actitudes positivas hacia el objeto de representación, indican que el apego, quizás excesivo, al manual escolar se resistirá al cambio. Estos resultados obtenidos llevan a proponer que para lograr un uso crítico del mismo, acorde con un posicionamiento pedagógico y didáctico que favorezca el interés de los estudiantes por aprender ciencias, es necesario destinar más tiempo a reflexionar sobre estos aspectos en la formación inicial, sosteniendo espacios en que se genere un uso reflexivo del manual escolar en torno a una propuesta de estrategias de lectura y de aprendizaje según las demandas de la sociedad. 


\section{Referencias}

Abric, Jean-Claude. (2001). Prácticas sociales y representaciones. México: Coyoacán.

Alzate Piedrahita, María Victoria, Arbeláez Gómez, Martha, Gómez Mendoza, Miguel Ángel, Romero Loaiza, Fernando y Gallon Bedoya, Humberto. (2005). El texto escolar y las mediaciones didácticas y cognitivas. Pereira: Papiro.

Banet, Enrique. (2007). Finalidades de la educación científica en secundaria: opinión del profesorado sobre la situación actual. Enseñanza de las ciencias, 25(1), 5-20. Recuperado de http://ddd.uab.cat/pub/edlc/02124521v25n1/02124521v25n1p5.pdf

Chiavone, Luciana. (2014). Representaciones sociales de la identidad profesional del Ingeniero en docentes de grado de la Facultad de Ingeniería de la UdelaR: posibles repercusiones en su práctica docente (Tesis para optar por el grado de maestría en Psicología Social). Universidad de la República. Montevideo, Uruguay. Recuperada de https://www.colibri.udelar.edu.uy/bitstream/123456789/4377/1/Chiavone\%20Turino,\%2 $\underline{\text { OLuciana.pdf }}$

Cortes Junior, Lailton Passos, Corio, Paola y Fernandez, Carmen. (julio, 2010). Representações Sociais: Contribuição desta teoria na pesquisa de formação de professores de química a partir do termo indutor Química Ambiental. Trabajo presentado en el XV Encontro Nacional de Ensino de Química (XV ENEQ). Brasília, DF, Brasil. Recuperado de http://www.sbq.org.br/eneq/xv/resumos/R0538-1.pdf

Cuéllar, Luigi, Pérez, Royman y Quintanilla, Mario. (2005). La propuesta de Ernest Rutherford en los libros de texto en Colombia: un análisis desde la historia de las ciencias y la visión de transposición didáctica en ellos. Enseñanza de las Ciencias, (Número Extra, VII Congreso), 1-6. Recuperado de http://ddd.uab.cat/pub/edlc/edlc a2005nEXTRA/edlc a2005nEXTRAp63proern.pdf

D'Andrea, Ana M. y Corral de Zurita, Nilda (septiembre, 2006). Representaciones sociales de formadores de formadores sobre el éxito y el fracaso académico y el buen y el mal estudiante. Trabajo presentado en la XIII Reunión de Comunicaciones Científicas y Tecnológicas, Corrientes, Argentina. Recuperado de http://200.45.54.140/unnevieja/Web/cyt/cyt2006/09-Educacion/2006-D-007.pdf

Furió, Carles, Vilches, Amparo, Guisasola, Jenaro y Romo, Víctor. (2001). Finalidades de la enseñanza de las ciencias en la secundaria obligatoria. ¿Alfabetización científica o preparación propedéutica? Enseñanza de las ciencias, 19(3), 365-376. Recuperado de http://ddd.uab.cat/pub/edlc/02124521v19n3/02124521v19n3p365.pdf

García, María B. y Moro, Lucrecia E. (2004). Revisión crítica de los libros de texto de ciencias naturales utilizados en las escuelas de enseñanza general básica. Educación química, $\quad$ 281-285. 3 Recuperado de http://educacionquimica.info/search volume.php?id revista $=77$

García-Carmona, Antonio. (2013). Educación científica y competencias docentes: Análisis de las reflexiones de futuros profesores de Física y Química. Revista Eureka sobre 
enseñanza y divulgación de las ciencias, 10(4), 552-567. Recuperado de https://dialnet.unirioja.es/servlet/articulo?codigo=4544345\&orden=1\&info=link

Gazali, Andrea. (2005). Los manuales de nivel medio. En Liliana Cubo de Severino (cood.), Los textos de la ciencia. Principales clases del discurso científico (pp. 337-356). Córdoba, Argentina: Comunicarte.

Graça, Margarida, Moreira, Marco Antonio y Caballero, Concesa. (2004). Representações sobre a matemática, seu ensino e aprendizagem: um estudo exploratório. Revista Investigações em Ensino de Ciências, 9(1), 37-93. Recuperado de http://www.if.ufrgs.br/public/ienci/artigos/Artigo ID110/v9 n1 a2004.pdf

Guerra Ramos, María Teresa y López Valentín, Dulce María. (2011). Las actividades incluidas en el libro de texto para la enseñanza de las ciencias naturales en sexto grado de primaria: análisis de objetivos, procedimientos y potencial para promover el aprendizaje. Revista mexicana de investigación educativa, 16(49), 441-470. Recuperado de http://www.scielo.org.mx/scielo.php?script=sci arttext\&pid=S1405$\underline{66662011000200006 \& \operatorname{lng}=e s \& \operatorname{lng}=e n}$

Guirado, Ana María, Mazzitelli, Claudia y Olivera, Adela. (2012). Las Representaciones de Futuros Docentes acerca del Aprendizaje de la Física y de la Química. Revista Electrónica Iberoamericana de Educación en Ciencias y Tecnología, 3 (2), 169-192. Recuperado http://www.exactas.unca.edu.ar/riecyt/VOL\%203\%20NUM\%202/Archivos\%20Digitales/ RieCyT\%20V3\%20N2\%20Set\%202012\%20Doc\%20-8-.pdf

Guirado, Ana María, Mazzitelli, Claudia y Olivera, Adela. (2013). Representaciones sociales y práctica docente: una experiencia con profesores de Física y de Química. Revista de Orientación Educacional, 27(51), 87-105. Recuperado de http://www.roe.cl/index.php/roe/article/viewFile/39/32

Ibaceta, Gricelda, Moscato, Mónica y Schnersch, Ana. (marzo, 2012). Análisis de las prácticas de enseñanza en ciencias naturales de maestros noveles del nivel primario egresados del Instituto de Formación Docente Continua de Bariloche. Trabajo presentado en el III Congreso Internacional de Profesorado Principiante e Inserción Profesional a la Docencia. Santiago, Chile.

Jodelet, Denisse. (1986). La representación social: fenómenos, concepto y teoría. En Serge Moscovici, Psicología social Volumen II (pp. 469-494). Barcelona: Paidós.

Jodelet, Denise. (2008). Social representations: The beautiful invention. Journal for the Theory of Social Behaviour, 38(4), 411-430. Recuperado de http://onlinelibrary.wiley.com/doi/10.1111/j.1468-5914.2008.00383.x/full

Lima Miranda, Camila. (2014). As representações sociais de licenciandos em química sobre "Ser Professor" (Tesis para optar por el grado de Mestre em Ensino de Ciências). Universidade de São Paulo, Brasil). Recuperado de http://www.teses.usp.br/teses/disponiveis/81/81132/tde-15012015154923/publico/Camila Lima Miranda.pdf 
Lotti de Santos, Margarita, Salim, Raquel, Raya, Francisco y Dori, María G. (2008). Una experiencia de formación docente sobre lectura comprensiva de textos científicos. Revista Iberoamericana de Educación, (45/3), 1-5. Recuperado de https://dialnet.unirioja.es/servlet/articulo?codigo=2554676\&orden=150552\&info=link

Marcelo, Carlos y Vaillant, Denise. (2009). Desarrollo profesional docente. Madrid: Narcea.

Martínez Losada, Cristina y García Barros, Susana. (2003). Las actividades de primaria y ESO incluidas en libros escolares. Enseñanza de las Ciencias, 21(2), 243-264. Recuperado de http://ddd.uab.cat/pub/edlc/02124521v21n2/02124521v21n2p243.pdf

Mazzitelli, Claudia. (2007). El aprendizaje de la Física como reelaboración conceptual a la luz de algunas teorías psicosociales (Tesis doctoral para optar por el grado de doctorado en Educación). Facultad de Filosofía y Letras, Universidad Nacional de Cuyo, Mendoza, Argentina.

Mazzitelli, Claudia. (2012). Representaciones acerca de la enseñanza y el aprendizaje de las Ciencias durante la formación docente inicial. Profesorado. Revista de Currículum y Formación de Profesorado, 16(3), 1-15. Recuperado de http://www.ugr.es/local/recfpro/rev163COL10.pdf

Mazzitelli, Claudia. (2013). Los futuros docentes y sus representaciones de la enseñanza de las ciencias. Avances en Ciencias e Ingeniería, 4(2), 99-110. Recuperado de https://dialnet.unirioja.es/descarga/articulo/4393463.pdf

Mazzitelli, Claudia. (2015). La enseñanza de las ciencias y la formación docente inicial: estudio de las representaciones sociales de estudiantes y docentes formadores. San Juan (Argentina): Universidad Nacional de San Juan.

Mazzitelli, Claudia, Aguilar, Susana, Guirado, Ana y Olivera, Adela. (2009). Representaciones sociales de los profesores sobre la docencia: contenido y estructura. Revista Educación, Lectura y Sociedad, 6 (6), 265-290. Recuperado de http://www.academia.edu/download/31068664/n06a14mazzitelli.pdf

Mazzitelli, Claudia, Guirado, Ana y Olivera, Adela. (febrero, 2010). Estudio de las representaciones acerca de la docencia en alumnos de profesorado. Implicancias para su futuro desempeño docente. Trabajo publicado en las Memorias del II Congreso Internacional sobre Profesorado Principiante e Inserción Profesional a la Docencia: El acompañamiento a los docentes noveles: prácticas y concepciones. Buenos Aires, Argentina. Recuperado de http://cedoc.infd.edu.ar/noveles/principiantes/5/INV 5 Mazzitelli ESTUDIO DE LAS REPRESENTACIONES.pdf

Melo, Énery, Tenório, Alexandro y Accioly, Horácio. (2010). Representações sociais de ciência de um grupo de licenciandos em Física. REEC: Revista Electrónica de Enseñanza de las Ciencias, 9(2), 457-466. Recuperado de http://reec.uvigo.es/volumenes/volumen9/ART9 Vol9 N2.pdf 
Mora, Martín. (2002). La teoría de las representaciones sociales de Serge Moscovici. Athenea digital: revista de pensamiento e investigación social, (2), 1-25. Recuperado de http://ddd.uab.cat/pub/athdig/15788946n2/15788946n2a8.pdf

Moscovici, Serge. (1979). El psicoanálisis, su imagen y su público. Buenos Aires: Huemul.

Petracci, Mónica y Kornblit, Ana Lía. (2007). Representaciones sociales: una teoría metodológicamente pluralista. En Ana Lía Kornblit (Coord.), Metodologías cualitativas: modelos y procedimientos de análisis (pp. 91-110). Buenos Aires: Editorial Biblos.

Piña Osorio, Juan y Cuevas Cajiga, Yazmín. (2004). La teoría de las representaciones sociales: Su uso en la investigación educativa en México. Perfiles educativos, 26(105106), 102-124. Recuperado de http://www.scielo.org.mx/scielo.php?pid=S018526982004000100005\&script=sci arttext

Ramírez, Tulio. (2003). El texto escolar: una línea de investigación en educación. Revista de $\begin{array}{llll}\text { Pedagogía, 24(70), 273-292. Recuperado de } & \end{array}$ http://www.scielo.org.ve/scielo.php?script=sci arttext\&pid=S079897922003000200003\&lng=es\&tlng=es

Rivas, José Ignacio y Leite, Analía. (2013). Aprender la profesión desde el pupitre. Cuadernos de Pedagogía, (436), 34-37. Recuperado de http://www.academia.edu/12339436/Aprender la profesi\%C3\%B3n desde el pupitre

Sancho, Juana, Hernández, Fernando, Martínez, Sandra, Padilla, Petry, Paulo, Montané López, Alexandra, Herraiz, García, Fernando, ...Losada, Daniel. (2014). La construcción de la identidad docente del profesorado de educación infantil y primaria en la formación inicial y los primeros años de trabajo (Identidoc). Síntesis y principales resultados y contribuciones. En Juana Sancho, José Correa, Xavier Giró y Leticia Fraga (Coord.), Aprender a ser docente en un mundo en cambio (pp. 11-23). Barcelona: Dipòsit Digital de la Universitat de Barcelona. Recuperado de http://som.esbrina.eu/aprender/docs/IDENTIDOC RESUMEN.pdf

Santos de Jesus, Tiago, Nunes Mota, Thaís R., Santos de Jesus, Weverton y Pagan, Acácio. (junio, 2011). A representações sociais de graduandos de ciências biologicas sobre ser professor. Trabajo presentado en el 4ํㅡㄹ Encontro de Formação de Professores e 0 5은 Fórum Permanente de Inovação Educacional - Edição Internacional. Universidade Tiradentes. Programa de Pós-Graduação em Educação (PPED/UNIT). Tiradentes, Brasil. Recuperado de http://www.unit.br/hotsites/2011/enc formacao professores/arquivos/artigos/GT 1 ES PACOS EDUCATIVOS/REPRESENTACOES SOCIAIS GRADUANDOS CIENCIAS BIOLOGICAS PROFESSOR.pdf

Solbes, Jordi, Montserrat, Rosa y Furió, Carles. (2007). Desinterés del alumnado hacia el aprendizaje de la ciencia: implicaciones en su enseñanza. Didáctica de las Ciencias Experimentales $y$ Sociales, (21), 91-117. Recuperado de http://cjis.uv.es/index.php/dces/article/download/2428/1973 
Solís Ramírez, Emilio, Luna Pérez, Manuel y Rivero García, Ana. (2002). Las concepciones y los problemas profesionales del profesorado "novel" de secundaria del área de ciencias de la naturaleza. Fuentes: Revista de la Facultad de Ciencias de la Educación, (4), 127-138. Recuperado

https://ojs.publius.us.es/ojs/index.php/fuentes/article/view/2432

Soliveres, María A., Maturano, Carla y Quiroga, Daniela. (octubre, 2014) ¿Qué actividades proponen los docentes de Ciencias Naturales para guiar la lectura de textos disciplinares? Trabajo publicado en las Actas del Congreso Nacional Cátedra UNESCO para el mejoramiento de la calidad y equidad de la educación en América Latina, con base en la lectura y la escritura: La lectura y la escritura en las sociedades del siglo XXI (Repositorio Universidad Nacional de Rosario). Rosario, Argentina. Recuperado de http://rephip.unr.edu.ar/handle/2133/4854

Torres Salas, María Isabel. (2010). La enseñanza tradicional de las ciencias versus las nuevas tendencias educativas. Revista Electrónica Educare, 14(1), 131-142. Recuperado

de http://revistas.una.ac.cr/index.php/EDUCARE/article/download/1515/1435

Tosi, Carolina. (2011). El texto escolar como objeto de análisis. Un recorrido a través de los estudios ideológicos, didácticos, editoriales y lingüísticos. Lenguaje, 39(2), 469-500. Recuperado de http://www.scielo.org.co/pdf/leng/v39n2/v39n2a08.pdf

Vaillant, Denise. (septiembre, 2007). La identidad docente. Trabajo presentado en el I Congreso Internacional Nuevas Tendencias en la Formación Permanente del Profesorado (GTD-PREALORT). Barcelona, España. Recuperado de http://www.ub.edu/obipd/docs/la identidad docente vaillant d.pdf

Wachelke, João y Wolter, Rafael. (2011). Critérios de construção e relato da análise prototípica para representações sociais. Psicologia: Teoria e Pesquisa, 27(4), 521-526. Recuperado de http://www.scielo.br/scielo.php?script=sci arttext\&pid=S0102$\underline{37722011000400017}$

Zorrilla, Erica y Mazzitelli, Claudia. (2014). Las representaciones sociales acerca de la Física: análisis de la influencia en el desempeño de futuros docentes. En Javier Maquilón Sánchez y José Alonso Roque (Comps.), Experiencias de innovación y formación en educación (pp. 45-52). Murcia: edit.um. 